\title{
MUTLI-ELEMENT SYSTEM RELIABILITY USING MARKOV CHAIN MODEL
}

Up to now our researches have focused on determining the existing bridge element reliability level. However, the real structures occurring in practice are not just one-element structures, but they are a set of multiple elements with various signification of single element in structure. On this account, current research activities were focused on determining multi-element structure reliability level. In the presented paper, the steel grid system with m-elements subjected to bending was considered. The Markov chain models were used to calculate structure reliability. The results are compared with analytic solution of system reliability. Analogously, the degradation of material properties and its influence on reliability of overall multi-element grid system was considered.

\section{Introduction}

In the frame of research activities of the Department of Structures and Bridges, the increased attention was concentrated on determining the reliability level for evaluation of existing bridge structures. The reliability level, which respects the positive influence of new information achieved by regular inspection of existing bridges, was determined using a probabilistic theory. The positive result of inspection is a condition for survival of a structure in the remaining lifetime. It means that any component of the structure has not exceeded any limit states until the inspection time. The reliability level depends on time of inspection and on a planned remaining lifetime of bridges [1], [2]. The values of a modified reliability level, which are expressed by a failure probability $P_{f t}$ resp. reliability index $\beta_{t}$, were used to specify partial safety factors for actions and material properties in the partial safety factors method.

Whereas the reliability level was deduced for one-element structure, nowadays, further possibility of reliability level specification considering multi-element system is observed. The model of multielement structures more correctly describes the real structures. In current approaches, the series or parallel systems or their mutual combinations are elaborated. Moreover, it is assumed that elements of a system fail independently of each other. The details can be found in [3]. However, the real bridge structure is not either one of these theoretical models in most cases, therefore, the well-turned formulation of a multi-element system reliability is found.

In the theoretical approach, the steel grid system with $m$-elements subjected to bending was considered. The reliability of the grid system is defined by Markov chains model, which describes the multi-element system preferably compared to analytic solution.

The basic idea of theoretical approach and the results of parametric study are presented in the paper. The influence of material degradation on the change of a multi-element system reliability level in time in comparison with one-element system is also taken into account.

\section{The reliability of a multi-element structure}

In the approach, the grid bridge structure consisting of $m$-elements is considered. Each of the elements has the resistance $R_{j}$ (for $j=1 \ldots \mathrm{m}$ ). It is assumed that the single resistances $R_{j}$ of the elements are independent normally distributed random variables $N\left(m_{j}, s_{j}\right)$, (for $\left.j=1 \ldots m\right)$. Moreover, the normally distributed random variables $N(\mu, \sigma)$ loads effects $S_{i}$ have effected on the bridge structure. The load effects $S_{i}$ occur in succession but randomly in time and their occurrence is considered as a random variable having Poisson distribution with parameter $\lambda(t), \lambda(t)>0$ for $t \in(0, T)$. The load is distributed to all system elements and the load effects $E_{i j}=a_{i j} \cdot S_{i}$ are induced. The following marginal conditions are valid for the factor $a_{i j}$

$$
-1,0 \leq a_{i j} \leq 1,0 \text {, and for a grid system } \sum_{j=1}^{m} a_{i j}=1,0
$$

shall be fulfilled.

The load effects $E_{i j}$ are normally distributed random variables with the mean value $\mu_{j}=a_{i j} \cdot \mu$ and the standard deviation $\sigma_{j}=$ $=a_{i j} \cdot \sigma$.

The failure probability $P_{f j}$ of $j$ element of the $m$-elements system subjected to a Poisson live load process with intensity $\lambda$ can be expressed according to [4] by a formula

$$
P_{f j}=\int_{-\infty}^{\infty} \phi\left(\frac{a_{i j} \cdot x-m_{j}}{s_{j}}\right) \cdot f_{\max }(x) d x .
$$

The failure probability $P_{f, j k}$, when the m-elements system fails due to the failure of element $\mathrm{j}$ together with element $k$, can be expressed by the relation

\footnotetext{
* Peter Koteš, Josef Vičan

Department of Structures and Bridges, Faculty of Civil Engineering, University of Žilina, Komenského 52, 01026 Žilina

Tel. +421-41-41868, Fax. +421-41-41868, E-mail kotes@ fstav.utc.sk, vican@fstav.utc.sk
} 


$$
P_{f, j k}=\int_{-\infty}^{\infty} \phi\left(\frac{a_{i j} \cdot x-m_{j}}{s_{j}}\right) \cdot \phi\left(\frac{a_{i k} \cdot x-m_{k}}{s_{k}}\right) \cdot f_{\max }(x) d x
$$

In the case of a failure of any system element, the failure probability $P_{f}$ of the system is expressed by a formula

$$
\begin{aligned}
P_{f}= & 1\left[\left(\int_{-\infty}^{\infty} \prod_{j=1}^{m}\left(1-\phi\left(\frac{a_{i j} \cdot x-m_{j}}{s_{j}}\right)\right) .\right.\right. \\
& \left.\left.\cdot f_{\max }(x) d x\right)+e^{-L(t)}\right],
\end{aligned}
$$

where $f_{\max }(x)=F_{\max }(x) \cdot \frac{L(T)}{\sigma} \cdot \varphi\left(\frac{x-\mu}{\sigma}\right)$,

and $F_{\text {max }}(x)=\sum_{n=0}^{\infty}\left[\phi\left(\frac{x-\mu}{\sigma}\right)\right]^{n} \cdot \frac{L(T)}{\sigma} \cdot e^{-\left(L(T)\left[1-\phi\left(\frac{x-\mu}{\sigma}\right)\right]\right)}$

The details of the above-described mathematical model can be found in [4] or [5]. Then, the reliability indices of any elements and whole system can be determined as follows

$$
\beta_{j}=-\phi^{-1}\left(P_{f j}\right) \text {, respect. } \beta=-\phi^{-1}\left(P_{f}\right) .
$$

The probability of no system failure due to any loads is also included in the relation (4).

\section{Dependent failure of elements}

In the standard approaches for multi-element systems it is assumed that the elements of a system fail independently of each other. It means that the failure of the one element does not influence the failure of another element. This incorrect assumption was demonstrated in paper [6]. Therefore, the approach using dependent failure of elements, which better describes the multi-element grid system, was found.

It was considered in the mathematical model that the failure of the system comes into being when the elements $j$ and $k$ fail, but in this case the elements of system fail dependently of each other. It means that the failure of one element influences the failure of another element. The five-element grid system was considered in the parametric study. This system represents the plate girder bridge subjected to bending.

The random variables resistances $R_{j}$ of the elements were considered as normally distributed $N\left(m_{j}, s_{j}\right)$ with parameters $m_{j}=$ $=159.66 \mathrm{MPa}$ and $s_{j}=12.993 \mathrm{MPa}$, for $j=1,2,3,4,5$.

The random variables load effects $S_{i}$ were also considered as normally distributed with parameters $\mu=125.50 \mathrm{MPa}$ and $\sigma=$ $=30.00 \mathrm{MPa}$. The load effects $S_{i}$ are divided to individual elements of the system by proportions $p_{i}(\%)$. The values of the proportions $p_{i}$ represent transversal load distribution, for which determination the model of rigid transversal beam was used. The lifetime of the system $T=80$ years and the constant parameter $\lambda(\tau)=0.0125$ of the failure rate were considered. It means that the most loaded element of the system was designed for a reliability level given by the reliability index $\beta_{j}=3.80$.

The problem with dependent failure of elements was solved by means of changing proportions $p_{i}$ of load effects on elements. The same load effects $S_{i}$ shall be carried by $m-1$ elements system after failure of one element without any changes of remaining elements resistances $R_{j}$ and any changes of load positions.

In the case of five-element system, the proportions $p_{i}$ are equal to (see Fig. 1 a) $p_{1}=60 \%, p_{2}=40 \%, p_{3}=20 \%, p_{4}=0 \%$ and $p_{5}=-20 \%$.

Then, it was considered that the most loaded element $\left(p_{1}=\right.$ $=60 \%$ - outer beam) had failed. New proportions pi were determined from the new four-element system (see Fig. 1 b) $p_{2}=100 \%$, $p_{3}=50 \%, p_{4}=0 \%$ and $p_{5}=-50 \%$

After failure of the next most loaded element (now, it is $p_{2}=$ $=100 \%$ - outer beam of the four-element system), new proportions pi were considered for the new three-element system (see Fig. $1 \mathrm{c}$ ) $p_{3}=183.333 \%, p_{4}=33.333 \%$ and $p_{5}=-116.667 \%$.

The results of the parametric study are presented in Tab. 1

The failure probability $P_{f}$ and the reliability

Tab. 1 index $\beta$ of the five-element system after failure first, second and third element.

\begin{tabular}{|c|c|c|}
\hline Failure of element & Failure probability $P_{f}$ & Reliability index $\beta$ \\
\hline 1 & $7.20269 \mathrm{E}-05$ & 3.801 \\
\hline 1 and 2 & $1.33782 \mathrm{E}-01$ & 1.109 \\
\hline 1,2 and 3 & $5.90448 \mathrm{E}-01$ & -0.228 \\
\hline
\end{tabular}

In the case of independent failure of elements, proportions $p_{i}$ of the load effects on elements are not changed, so the second most loaded element takes just $40 \%\left(p_{2}=40 \%\right)$ from the loads (see Fig. 1 a). Then using the formula (3), the failure probability of the system is influenced by the failure probability of the second most load element and it is equal to $P_{f}=1.10^{-12}[6]$. Such probability of failure is unrealistic for real structures.

From the results given in Tab. 1 using the dependent failure of elements, it can be seen that the reliability level is influenced by the most loaded element (the most faithless element) of the fiveelement system. After its failure, the reliability level of the system given by the reliability index $\beta$ strongly decreases $(\beta=3.80 \rightarrow$ $\beta=1.11-$ difference is about $71 \%$ ). So big loss of the reliability level and the failure of the most loaded element are inadmissible for real grid structures. Moreover, the failure of the next element decreases the reliability level to $\beta=-0.23$ corresponding to the failure probability of $P_{f}=0.59$.

However, the disadvantage of the analytic solution is the fact that the failure of two or more elements given by the formula (3) is able to come into being only together. But this failure in reality 


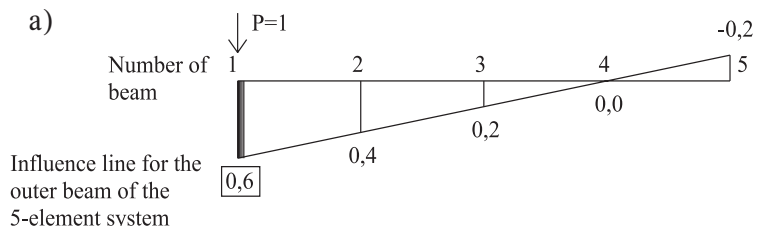

5-element system

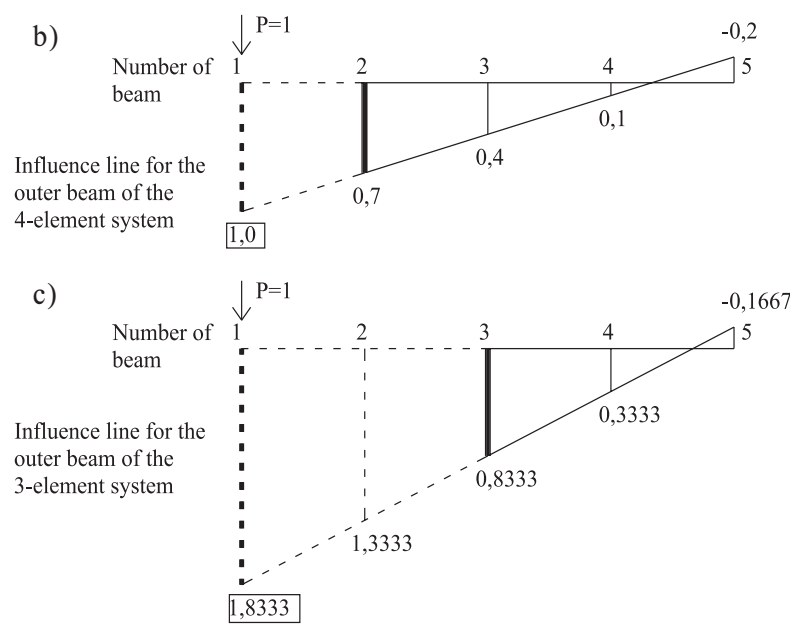

Fig. 1 Dependent failure of five-element system - successive changing load distribution after failure of the elements

is able to come into being by steps - the first element fails and then the second element is able to fail, not just together. Therefore, a more correct approach to define the reliability level or the failure probability of m-element system was found.

\section{Approach using homogeneous Markov chains model}

The multi-element system is observed at time steps (periods) $k=1,2,3 \ldots$. The system is assumed to have $(r+1)$ possible states numbered $j=0,1,2 \ldots r$. The system is in state $i$ at time $k$ with probability $p_{k}(i)$. Many systems have transitions that can be approximately described by a stochastic process with the Markov property. If the probability $p_{k}(i)$ depends just on the state in time $k-1$ (not on states in time $k-2, k-3, \ldots$ ) for every state $i$, the system is called Markov chains. Given that a system is in state $i$ at time $k$, the future states $(i+v)$ do not depend on the previous states $(u<i)$. In other words, when its present state is known, the probability of any particular future behaviour of the process is not altered by additional knowledge about its past behaviour. Moreover, $P(i, j)=p(i, j)$ is the transition probability that the system is in state $j$ at time $t$ if the system was in state $i$ at time $(t-1)$. It is homogeneous Markov chain model, if the probability $P(i, j)$ does not depend on time $t$. The matrix of array $P(i, j)$, for $i, j=0,1, \ldots r$, is called the matrix of homogeneous system.

Next, $P_{m}(i, j)$, for $i, j=0,1, \ldots r$, is the probability that the Markov chain is in time $t+m$ in state $j$ if it was in time $t$ in state $i$ (do not depend on time $t$ ). So, $P_{m}(i, j)$ is called as the transition matrix after $\mathrm{m}$-steps and following relation is valid

$$
\begin{aligned}
& P_{m}(i, j)=\sum_{k=1}^{r} P_{m-1}(i, j) \cdot P(k, j)=\sum_{k=1}^{r} P(i, j) \cdot P_{m-1}(k, j), \\
& \text { for } i, j=0,1, \ldots r .
\end{aligned}
$$

Owing to a successive institution it can be seen that

$P_{m}(i, j)=P^{m}(i, j)$, it means m-exponent of transition matrix $P(i, j)$.

Firstly, $m$-element system without degradation was considered in the approach. One year was given as a time step. The states of the system were determined by a number of possible failed elements with respect of their location and their significance in the structures. It means $m+1$ states for m-element system. The transition probabilities between individual states can be solved analytically using the formulae (2), (3) and (4). So, the development of the probabilities of single states in time as a chosen member of the transition matrix $P_{m}(i, j)$ after m-steps is observed.

In the parametric study, the same parameters as in the analytic solution were used.

The values of probabilities for lifetime $\mathrm{T}$ are achieved by multiplying the transition matrices. The results of the parametric study are shown in Fig. 2 and in Fig. 3. The reliability index development of the most loaded element in time $t$ is shown in Fig. 2 in comparison with analytic solution and time development of the same single element reliability. The reliability index development of the second most loaded element of the five-element system in time $t$ is shown in Fig. 3 in comparison with an analytic solution.

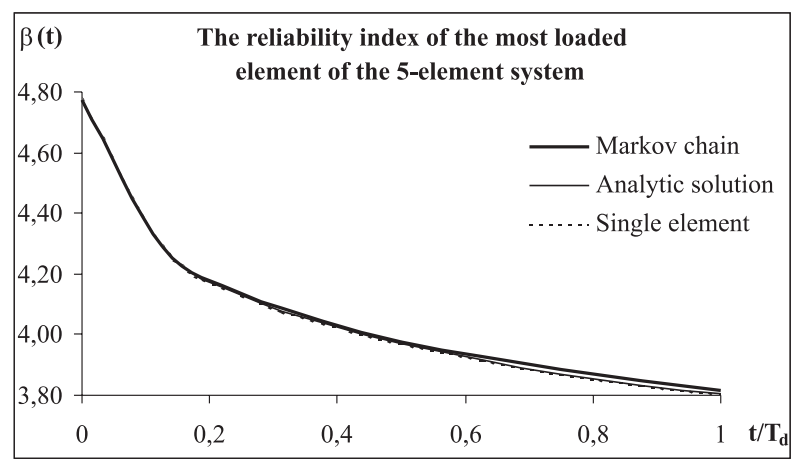

Fig. 2 The change of the most loaded element reliability index of the five-element system in time

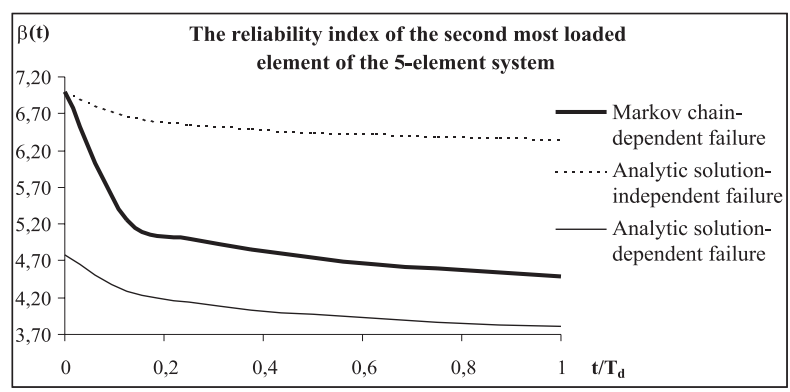

Fig. 3 The change of second element reliability index of the five-element system in time 
From the achieved results it can be seen that the Markov chains model describes the real system reliability more appropriately due to considering the dependent failure of elements (and the failure of elements) in stages (Fig. 3). Moreover, it can be seen that the reliability level of the system is significantly influenced by reliability of the most loaded element (the most faithless element) so that the element could be investigated without considering the system (Fig. 2).

\section{Approach using inhomogeneous Markov chains model}

If the probability $P(i, j)$ depends on time $t$, the process is called inhomogeneous Markov chain model and the denotation $P(i, j)=$ $=P_{t}(i, j)$ can be established. The inhomogenity of the transition between stages can be caused by degradation of material. The resistance $R_{j}(t)$ changes in time using material degradation, so, the transition probability given by the formulae (2), (3) and (4) is also change in time. On this account, the transition matrix $P(i, j)$ is not constant in time between stages, but these transition matrices between single stages have to be solved in particular time. Now, the transition matrix after m-steps $P_{m}(i, j)$ is achieved by multiplying the matrices $P_{t}(i, j)$.

The steel grid five-element system was considered in the parametric study. The corrosion of the I-steel beams flanges was considered as degradation of the material and the resistance $R_{j}(t)$. The corrosion is a time dependent factor that influences not only resistance $R_{j}(t)$, but also influences the flexural stiffness and the redistribution of the moments over the system. The corrosion model of Albrecht and Naeemi [7], which was successfully applied in works [8], [9], given by the formula

$$
d_{\text {corr }}=A_{0} \cdot t^{A_{1}} .
$$

was used.

The dependence (10) was estimated by mathematical approximation of values, which were achieved by measurement of flange thickness decrease in case of the real steel bridge I - beams. The constants $A_{0}, A_{1}$ take into account the location of the beams in the grid system and the following values were recommended

1. $A_{0}=0.13218, A_{1}=0.595478$ for the outer girder of the grid,

2. $A_{0}=0.12151, A_{1}=0.568652$ for the second outer girder of the grid,

3. $A_{0}=0.03015, A_{1}=0.690171$ for the internal girder of the grid

The geometric and material parameters are considered as random variables quantities and the denotation of the geometric parameters are shown in Fig. 4. The values of random variables are shown in Tab. 2

Time dependent resistance $R_{j}(t)$ of the elements subjected to bending is given by the following formulae

$R_{j}(t)=f_{y} \cdot W(t)_{p l y}$, for cross-section of the class 1 and 2 ,

$R_{j}(t)=f_{y} \cdot W(t)_{e l, y}$, for cross-section of the class 3 ,
Values of random variables

Table 2

\begin{tabular}{|c|c|c|c|}
\hline \multirow{2}{*}{ Variable } & \multicolumn{2}{|c|}{ I 800} & \multirow{2}{*}{ istribution function } \\
\cline { 2 - 3 } & $\mathrm{m}$ & $\mathrm{s}$ & \\
\hline $\mathrm{f}_{\mathrm{y}}[\mathrm{MPa}]$ & 281.00 & 27.80 & Empirical \\
\hline $\mathrm{b}_{\mathrm{f}}[\mathrm{mm}]$ & 250.00 & 2.00 & Normal. \\
\hline $\mathrm{t}_{\mathrm{f}}[\mathrm{mm}]$ & 25.525 & 0.925 & Normal \\
\hline $\mathrm{d}[\mathrm{mm}]$ & 750.00 & 2.00 & Normal \\
\hline $\mathrm{t}_{\mathrm{w}}[\mathrm{mm}]$ & 10.210 & 0.37 & Normal \\
\hline
\end{tabular}

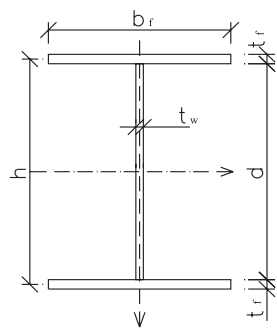

Fig. 4 Geometrical parameters of the observed cross-section

where $f_{y} \quad$ is the random variable yield strength,

$W(t)_{p l, y}$ is random variable time dependent plastic section modulus,

$W(t)_{e l, y}$ is random variable time dependent elastic section modulus.

Time dependent plastic section modulus for cross-section of class 1 or 2 taking into account the corrosion of the flanges is described by the formula

$$
\begin{aligned}
W(t)_{p l, y} & =0,25 \cdot t_{w} \cdot\left(d+A_{0} \cdot t^{A_{1}}\right)^{2}+ \\
& +b_{f} \cdot h \cdot\left(t_{f}-A_{0} \cdot t^{A_{1}}\right),
\end{aligned}
$$

and similarly, time dependent elastic section modulus for crosssection of class 3 taking into account the corrosion of the flanges is describ-ed by the following formula

$$
\begin{aligned}
W(t)_{e l, y}= & {\left[\frac{t_{w} \cdot\left(d+A_{0} \cdot t^{A_{1}}\right)^{2}}{6}+b_{f} \cdot h^{2} \cdot\left(t_{f}-A_{0} \cdot t^{A_{1}}\right)\right] / } \\
& /\left[\mathrm{d}+2 \cdot\left(t_{f}-A_{0} \cdot t^{A_{1}}\right)\right],
\end{aligned}
$$

The numerical application of the above-mentioned process of time dependent resistance calculation considering steel T-beam flanges corrosion was carried out using simulation by Monte Carlo method. For other usage the results of simulation were approximated by mathematical relations

$m_{R}(t)=m_{R}\left(t_{0}\right) \cdot e^{p_{1} \cdot t^{\prime 2}}$, for the mean value of resistance

$s_{R}(t)=s_{R}\left(t_{0}\right) \cdot e^{p_{3} \cdot t^{\prime 4}}$, for the standard deviation of resistance (16)

where $m_{R}\left(t_{0}\right), s_{R}\left(t_{0}\right)$ are the mean value and the standard deviation of resistance in time $t=0$,

$p_{1}, p_{2}, p_{3}, p_{4}$ are constants achieved by mathematical approximation. 


\begin{tabular}{|c|c|c|c|c|c|c|}
\hline \multirow{2}{*}{ I-beam } & \multicolumn{6}{|c|}{ Parameters } \\
\cline { 2 - 7 } & $m_{R}\left(t_{0}\right)$ & $p_{1}$ & $p_{2}$ & $s_{R}\left(t_{0}\right)$ & $p_{3}$ & $p_{4}$ \\
\hline I $800-1(\mathrm{~A} 0, \mathrm{~A} 1)$ & 1792.906 & -0.00347 & 0.63309 & 186.036 & -0.00205 & 0.72949 \\
\hline I $800-2(\mathrm{~A} 0, \mathrm{~A} 1)$ & 1792.906 & -0.00314 & 0.60814 & 186.036 & -0.00169 & 0.72502 \\
\hline I $800-3(\mathrm{~A} 0, \mathrm{~A} 1)$ & 1792.906 & -0.00055 & 0.80400 & 186.036 & -0.00003 & 1.49558 \\
\hline
\end{tabular}

The values of constants $p_{1}, p_{2}, p_{3}, p_{4}$ and $m_{R}\left(t_{0}\right), s_{R}\left(t_{0}\right)$ for I-beams taking into account the location of beam in grid system given by constants $A_{0}, A_{1}$ are shown in Tab. 3 .

Time dependent resistance given by (11) or (12) described by the mean value and the standard deviation enters into relations (2), (3) and (4). Now, it is able to solve the transition probabili-

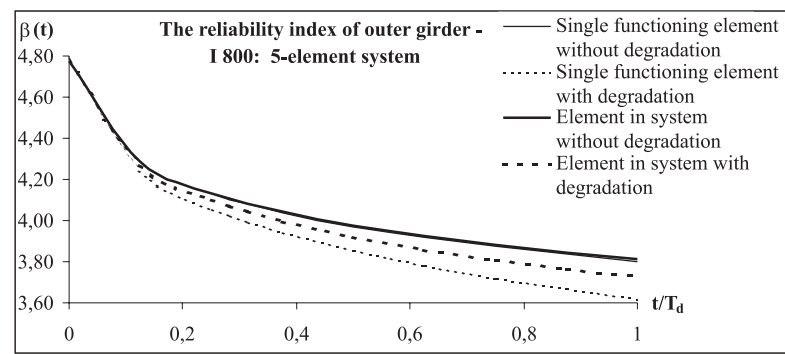

Fig. 5 The change of reliability index of the element in time

ties $P_{t}(i, j)$ depending on time steps due to degradation of resistance. The transition matrix after m-steps $P_{m}(i, j)$ is achieved by multiplying the different matrices $P_{t}(i, j)$.

The comparison of the results achieved by considering the same element (outer girder of the grid) single functioning and in the five-element system and considering corrosion or without corrosion is shown in Fig. 5.

\section{Conclusions}

From the results it can be seen that the reliability level of the system is significantly influenced by the most loaded element (generally, it is the outer girder of the grid system). Therefore, the element can be investigated without considering the system if corrosion is neglected. It means that we can focus just on one most loaded element in the system and investigate it without considering the whole system (see Fig. 2). On this account, the ultimate limit state of the overall system is defined by a failure of the most loaded element However, if the corrosion of steel I-beam flanges is considered, the new lower reliability indices are achieved (Fig. 5). By comparison of the result, when the element under corrosion is considered and investigated in the system, the element has a higher reliability level $(\beta=3.73)$ at the end of lifetime $T$ against the same element considered as just single functioning ( $\beta=3.62$ ). The difference is about $9.6 \%$. This difference was achieved by changing flexural stiffness and the redistribution of the actions over the system.

In the case of an element with degradation, it is more correct to investigate the element in the system due to a possible higher reliability level of the element and the overall system. The conclusion is very important from the viewpoint of a reliability-based evaluation of existing structures where the effects of degradation are much more significant.

\section{Acknowledgements}

The research work presented in this paper has been supported by the Slovak Grant Agency, Grant No. 1/0344/03.

\section{References}

[1] KOTEŠ, P., VIČAN, J.: Reliability of Concrete Structure Members under Corrosion Attack. Proceedings of a 19-th International conØference 'Durability Design and Fracture Mechanics of Concrete Structures', Minsk, 2003, 38-46.

[2] VIČAN, J., KOTEŠ, P.: To Evaluation of existing Railway Bridges. Inžinierske stavby, roč. 50, 2002, č.4, str. 19-22.

[3] HØYLAND, A., RAUSAND, M.: System reliability theory - Models and statistical methods. John Wiley and sons., 1994.

[4] KOTEŠ, P., SLAVÍK, J., VIČAN, J.: Reliability of multi-element bridge system. Proceedings of VII. Scientific Conference with International Participation, Košice 2002, p. 121-124.

[5] VIČAN, J., SLAVÍK, J.: Reliability level differentiation for existing bridge member. Proceedings of International Symposium "Theoretical and Technological Problems of Steel Structures”, Bratislava 1999, p. 151-156.

[6] KOTEŠ, P., SLAVÍK, J., VIČAN, J.: Multi-element bridge systems reliability. Proceedings of International Conference "Reliability and diagnostics of transport structures and means", Pardubice 2002, p. 173-179.

[7] ALBRECHT, P. - NAEEMI, A. H.: Performance of Weathering Steel in Bridges. National Cooperative Highway Research Program, Report 272, 1984.

[8] ESTES, A. C. - FRANGOPOL, D. M.: System Reliability for Condition Evaluation of Bridges. IABSE Workshop "Evaluation of Existing Steel and Composite Bridges", Lausanne 1987, p. 47-56.

[9] FRANGOPOL, D. M. - ESTES, A. C.: Lifetime Bridge Maintenance Strategies Based on System Reliability. Structural Engineering International 3, 1997, p. 193-198. 